\title{
Guaranteed Convergence Particle Swarm Optimization using Personal Best
}

\author{
1.Pawan Kumar Patel \\ Indian Institute of Technology Kanpur India
}

\author{
2.Vivek Sharma
}

MNNIT Allahabad, India

\author{
3.Kunal gupta \\ HCT,Muscat, Oman
}

\begin{abstract}
Particle Swarm Optimization (PSO), is well known technique for population based global search but its limitation to premature convergence before finding the true global minimiser. In this paper We introduce a technique by adding new parameters and a new velocity update formula using personal best value discovered by the swarm particles and decreasing the diameter of search space which prevents premature convergence before finding the true global minimiser. The resulting particle swarmoptimization (PGCPSO) provides a mechanism which is more efficient in finding true global minimizer while it was tested across the benchmark suite .

\section{KEYWORDS}

Particle Swarm Optimization,
\end{abstract}

\section{INTRODUCTION}

The term optimization refers to the study of problems in which one seeks to minimize or maximize a real function by systematically choosing the values of real or integer variables from within an allowed set. On one hand, a vast amount of research has been conducted in this area of knowledge with the hope of inventing an effective and efficient optimization algorithm. On the other hand, the application ofexisting algorithms to real projects has also been the focus of much research. The most commonly used optimization technique known as evolutionary computation (EC)[8]. Broadly speaking, EC constitutes a generic population-based metaheuristicoptimization algorithm. Evolutionary algorithms tend to perform well with regard to most optimization problems. This is the case because they refrain from simplifying or making assumptions about the original form. As a newly developed subset of EC, the Particle Swarm Optimization hasdemonstrated its many advantages and robust nature in recent decades. It is derived from the social behaviour of bird flocks in particular. Inspired by the swarm intelligence theory,Kennedy created a model which Eberhart then extended to formulate the practical optimization methodknown as particle swarm optimization (PSO) [1]. The algorithm behind PSO is based on the idea that individuals are able to evolve by exchanging information with their neighbours through social interaction. This is known as cognitive ability.

Three features impact on the evolution of the individual: Inertia (velocities cannot be changed abruptly), the individual itself (the individual could go back to the best solution found so far) and social influences (the individual could imitate the best solution found in its neighbour). PSO has a very common problem of stagnation and premature convergence, especiallyin multi modal function[7]. A lot ofresearch work have been done to improve the convergence behaviour of PSO. With this motivation we have tried to invent a new

variant of particle swarm optimization using the concept of Guaranteed Convergence Particle Swarm Optimization (GCPSO) [2] proposed by Van Der Bergh in 2002 which yields much better result than GCPSO.There is still a problem in GCPSO[3], however, in that particles tend to converge to a local minimizer before encountering a true global minimizer due to lack of exploration.

Addressing this problem, Van den Bergh[6] developed multi-start PSO (MPSO) whichautomatically triggers a restart when stagnation is detected. Various criteria for detecting premature convergence were tested in order to avoid the undesirable state of stagnation. It was thought that restarting on the original search space might cause unnecessarily repetitious searching of regions not expected to contain quality solutions. GCPSO might even allow the swarm to escape local optima ifparameters were designed with exploratory intentions, but this approach would effectivelyleave the rest of the swarm trailing almost linearly behind the globally best particles random movements, which would not be ideal. So a mechanism known to be regrouping PSO(RegPSO)[4] became desirable by which the swarm could efficiently regroup in a region small enough to avoid unnecessarily redundant search, yet large enough to escape wells containing local minima in order to try to prevent stagnation while retaining memory of only one global best rather than a history ofthe best of them. Consequently, there is one continuous search with each groupingmaking use of previous information rather than a series of independent searches.It produced better results than it's previous versions but this approach also lacks the exploration ability within the particles so to deal with this problem we propose an approach called guaranteed convergence PSO(PGCPSO) which could increase the exploration ability of particles while keeping the convergence rate fast and keeping a common approach of escaping local minima.

A roadmap of this paper is as follows. Related work is discussed in Section 2. A novel variant of GCPSOcalled PGCPSO is presented in Secton 3. Experimental results from Benchmark functions is discussed in Section 4. Section 5 covers the conclusion.

\section{Related Work}

PSO was introduced by Kennedy and Eberhart [1]. The behaviour of PSO can be envisioned by comparing it to bird swarms searching for optimal food sources, where the direction in which a bird moves is influenced by its current movement, the best food source it ever experienced, and the best food source any bird in the swarm ever experienced. In other words, birds are driven by their inertia, their personalknowledge, and the knowledge of the swarm. In terms of PSO, the movement of a particle is influenced by its inertia, its personal best position, and the global best position. PSO has multiple particles, and every particle consists of its current objective value, its position, its velocity, its personal best value, that is the best objective value the particle ever experienced, and its personal best position, that is the position at which the personal best value has been found. In addition, PSO maintains the global best value, that is the best objective value any particle has ever experienced, and theglobal best position, that is the position at which the global best value has been found. Classical PSO uses the following iteration to move the particle:

$x_{(i)}(t+1)=x_{(i)}(t)+v_{(i)}(t+1)$ 
$\mathrm{x}(\mathrm{i})$; is the position of particle $\mathrm{i}$, at a timestamp $\mathrm{t}$, and $\mathrm{v}_{(\mathrm{i})}$; is the velocity of particle i. In classical PSO, the velocity of the particle is determined using the following iteration:

$v_{i ; j}(t+1)=v_{i}(t)+c_{1} r_{1 ; j}(t)\left[p_{\text {best }}-x_{i ; j}\right]+c_{2} r_{2 ; j}\left[g_{\text {best }}-x_{i ; j}(t)\right]$

$\mathrm{p}_{\text {best }}$ is the best value of an individual particle and $\mathrm{g}_{\text {best }}$ is the global best position and $c_{1}$ and $c_{2}$ are the constant with value which is equal to 2 whereas $r_{1}$ and $r_{2}$ are random variables.PSO can focus on either convergence or diversity at any iteration. To focus on diversity means particles are scattered, searching a large area coarsely. To focus on convergence means particles are close to each other, searching a small area intensively. A promising strategy is to focus on diversity in early iterations and convergence in later iterations.

PSO has two major drawbacks. The first drawback of PSO is its premature character, i.e. it could converge to local minimum. Although PSO converges to an optimum much faster than other evolutionary algorithms, it usually cannot improvethe quality of the solutions as the number of iterations is increased. PSO usually suffers from premature convergence when high multi-modal problems are being optimized. The main reason is that for the standard PSO particles converge to a single point which is on the line connecting the global best and the personal best positions. Nevertheless this point is not guaranteed to be a local optimum and may be calledequilibrium point. The second drawback is that the PSO has a problem-dependent performance. This dependency is usually caused by the way parameters are set, i.e. assigning different parameter settings to PSO will result in high performance variance. After the PSO was issued, several considerations has been taken into account to facilitate the convergence and prevent an "explosion" of the swarm. These considerations focus on limiting the maximum velocity, selecting acceleration constants, constriction factor etc.

\subsection{GCPSO: Guaranteed Convergence PSO}

The basic idea of GCPSO[3] is to introduce an additional particle, which searches the region around the current global best position, i.e. its local best position is equal to the current global best position. In that manner, the current global best particle is treated also as a member of the swarm(e.g. particle ), the update formula for this particle is seen below:

$v^{\varphi}(t+1)=x^{\varphi}(t)+g_{\text {best }}(t)+\omega v^{\varphi}(t)+\rho(t)(1-2 r)$

It is noted that this variant is so far only applied to the $\mathrm{G}_{\text {best }}$ mode. The other particles in the swarm continue to use the normal velocity update formula, e.g. formula (3). Here the term $x^{\varphi}(t)+g_{\text {best }}(t)$ looks like the global cognitive part in formula (3). Because the global best position and the individual best position are coincident. $\rho(t)(1-2 r)$ substitutes the "social" part of the formula (3) to increase its search ability, which causes the additional particle to perform a random search in an area surrounding the global best position. Here $r$ is a vector randomly generated in the domain $[0,1]$ and $\rho(t)$ is the diameter of the search area and dynamicallyadapted based on the behaviour of the swarm, i.e. if the swarm always finds a better position than the current global best position in consecutive iterations, the search diameter will become larger, if the swarm always fails to find a better position than the current global best position in consecutive iterations, the search diameter willbecome smaller. The update formula of the diameter is as follows: $\rho(\mathrm{t}+1)=\left\{\begin{array}{lr}2 p(t) & \text { \#successes }>s c \\ \left(\frac{1}{1.5}\right) p(t) & \text { \#failures }>f c \\ p(t) & \text { otherwise }\end{array}\right.$

Volume 73-No.7, July 2013

Where terms \#successes and \#failures are defined as the number of the consecutive successes or failures, respectively, and the definition of failure is . The threshold parameters sc and fc are defined empirically. Since in a high dimensional search space, it is difficult to obtain a better value in only a few iterations, thus recommended values are thus $\mathrm{sc}=15$ and $\mathrm{fc}=5$. On some benchmark tests, the GCPSOhas shown a nice performance of locating the minimal of a unimodal function withonly a small amount of particles. This implies that it does not need many particlesto facilitate a local search. Compared with the original PSO, it also has a fasterconvergence on unimodal functions. This approach is a good supplement to theoriginal PSO, however it has a small aw, i.e. sometimes if the current global bestposition is located on a local minimum which is not near the global minimum, theadditional particle could possibly fail to find any better position around this localminimum and the algorithm would converge to the local minimum eventually.

\section{Our ApproachGCPSO Using Personal Best: PGCPSO}

The goal of the proposed GCPSO using personal best (GCPSO) is to not to limit the exploration ability to the few particles but to provide the opportunity to the other particles in the swarm so that they can escape from the local minimum. InPGCPSO an additional particle $(\varphi)$ similar to GCPSO is introduced which searches the region around the current personal best position, In that manner, the current update formula for this particle is seen below:

$v^{\varphi}(t+1)=x^{\varphi}(t)+p_{\text {best }}(t)+w v^{\varphi}(t)+\rho(t)(1-2 r)$

The other particles in the swarms are updated as per the formula introduced in PBPPSO

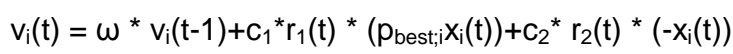

Here the term $-\mathrm{x}^{\varphi}(\mathrm{t})+\mathrm{p}_{\text {best }}(\mathrm{t})$ gives the particle ability to search as per their personal best position. $\rho(t)(1-2 r)$ substitutes the "social" part of the formula (4) to increase its search ability, which causes the additional particle to perform random search in an area surrounding the global best position. Here $r$ is a vector randomly generated in the domain $[0,1]$ and $\rho(t)$ is the diameter of the search area and dynamically adapted based on the behaviour of the swarm, i.e. if the swarm always finds a better position than the current global best position in consecutive iterations, the search diameter will become larger, if the swarm always fails to find a betterposition than the current global best position in consecutive iterations, the search diameter will become smaller. The update formula of the diameter is as follows:

$\rho(t+1)=\left\{\begin{array}{lr}2 p(t) & \text { \#successes }>s c \\ \left(\frac{1}{1.5}\right) p(t) & \# \text { failures }>f c \\ p(t) & \text { otherwise }\end{array}\right.$ 
Where terms \#successes and \#failures are defined as the number of the consecutive successes or failures, respectively, and the definition of failure is. The threshold parameters $\mathrm{sc}$ and $\mathrm{fc}$ are defined empirically. Since in a high dimensionalsearch space, it is difficult to obtain a better value in only a few iterations, thus recommended values are thus $\mathrm{sc}=15$ and $\mathrm{fc}=5$. Whereas the equation (4) gives the liberty to the other particles which are not best to search the space in theirrespective direction of personal best and if any of the particles evaluates with the better value than the previously defined best particle then that particle is replaced with the best particle and the search is concentrated as per the new value found.

All other previous versions of PSO performed well in unimodal function but when it's the case of multimodal function their efficacy was not upto the expectation because in previous versions all the particles didn't had the opportunity infinding the global minimum as it was limited to few best particles. In PGCPSO equation(4) leads the other particles in escaping the local minima as the values lead ny the best particle may not always lead to the global minima so it becomes necessary to provide the other particles with the exploration ability in their defined search space and direction to avoid premature convergence and stagnation. In casestagnation or premature convergence occurs in PGCPSO it can be resolved using MPSO or RegPSO as PGCPSO is not totally free from premature convergence.

\section{Result and analysis}

In this papertested that has been used to authenticate the proposed PGCPSO algorithm consists of five benchmark functions[9].Ackley function, Rastrigin function, Spherical function, Quartic function, and Griewenk function are the benchmark functions.

Table 1. Benchmark Function

\begin{tabular}{|c|c|c|}
\hline Function & Mathematical Representation & Range \\
\hline Ackley & $\begin{array}{c}\mathrm{f}(\mathrm{x})=-20 * \exp (- \\
\left.0.2 \sqrt{1 / n} \sum_{i=1}^{n} x_{i}^{2}\right)- \\
\exp \left(\sqrt{1 / n} \sum_{i=1}^{n} \cos \left(2 \pi x_{i}\right)\right)+ \\
20+e\end{array}$ & $\begin{array}{c}-32.0 \\
32.0]\end{array}$ \\
\hline Rastrigin & $\begin{array}{c}\mathrm{f}(\mathrm{x})=\sum_{i=1}^{n}\left[x_{i}^{2}-10 \cos \left(2 \pi x_{i}\right)+\right. \\
10]\end{array}$ & $\begin{array}{c}{[-10.0} \\
10.0]\end{array}$ \\
\hline Griewenk & $\begin{array}{l}\mathrm{f}(\mathrm{x})=\sum_{i=1}^{n}\left[\frac{x_{i}^{2}}{4000}-\right. \\
i=1 n \cos x i 2 i+1]\end{array}$ & $\begin{array}{c}{[-600.0,} \\
600.0]\end{array}$ \\
\hline Quartic & $\mathrm{f}(\mathrm{x})=\sum_{i=1}^{n}\left[i x_{i}^{2}+\operatorname{rand}[0,1)\right]$ & $\begin{array}{c}-1.28, \\
1.28] \\
\end{array}$ \\
\hline Spherical & $\mathrm{f}(\mathrm{x})=\sum_{i=1}^{n} x_{i}^{2}$ & $\begin{array}{c}{[-100.0} \\
100.0]\end{array}$ \\
\hline
\end{tabular}

Table 2. Benchmark Functions with their Global Minimum

\begin{tabular}{|c|c|}
\hline Function & Global Minimum \\
\hline Ackley & $\mathrm{f}\left(\mathrm{x}^{*}\right)=0$, at $\mathrm{x}=0$ \\
\hline Rastrigin & $\mathrm{f}\left(\mathrm{x}^{*}\right)=0$, at $\mathrm{x}=0$ \\
\hline Griewenk & $\mathrm{f}\left(\mathrm{x}^{*}\right)=0$, at $\mathrm{x}=0$ \\
\hline Quartic & $\mathrm{f}\left(\mathrm{x}^{*}\right)=0$, at $\mathrm{x}=0$ \\
\hline Spherical & $\mathrm{f}\left(\mathrm{x}^{*}\right)=0$, at $\mathrm{x}=0$ \\
\hline
\end{tabular}

Results implemented on different standard function is given in table 1. To make a fair comparisons parameters used are same for every benchmark functions and everyversions of PSO i.e particles 35, inertial weight $\omega=.73864$, for 200 iterations and velocity clamping upto $15 \%$ and $\mathrm{c} 1$ and $\mathrm{c} 2=1.49618[5]$. The results from the Table 1 . clearly indicate that PGCPSO is a better option than any of the other three. Mostalgorithm perform well in case of unimodal functions but they fail to provide a better result for multi-modal functions but from the table it can be clearly observed that PGCPSO also provides a good solution for multimodal benchmark functionthan the comparison algorithms due to it's better exploration capability in multi - modal functions than compared to other algorithms. 
Table 3. Result Table

\begin{tabular}{|c|c|c|c|c|c|c|c|c|}
\hline Function & \multicolumn{2}{|c|}{ PSO } & \multicolumn{2}{|c|}{ GCPSO } & \multicolumn{2}{|c|}{ RegPSO } & \multicolumn{2}{|c|}{ PGCPSO } \\
\hline Ackley & $\begin{array}{l}\text { Mean } \\
\text { Max } \\
\text { Median } \\
\text { Std.dev } \\
\end{array}$ & $\begin{array}{c}1.543326 \\
3.290502 \\
1.35123 \\
1.332453 \\
\end{array}$ & $\begin{array}{l}\text { Mean } \\
\text { Max } \\
\text { Median } \\
\text { Std.dev } \\
\end{array}$ & $\begin{array}{c}2.8635 \mathrm{e}-2 \\
2.0606 \mathrm{e}-5 \\
2.1316 \mathrm{e}-2 \\
1.3631 \mathrm{e}-10 \\
\end{array}$ & $\begin{array}{l}\text { Mean } \\
\text { Max } \\
\text { Median } \\
\text { Std.dev }\end{array}$ & $\begin{array}{l}4.6915 \mathrm{e}-7 \\
8.7023 \mathrm{e}-3 \\
4.4632 \mathrm{e}-7 \\
1.4519 \mathrm{e}-7 \\
\end{array}$ & $\begin{array}{l}\text { Mean } \\
\text { Max } \\
\text { Median } \\
\text { Std.dev } \\
\end{array}$ & $\begin{array}{l}1.0516 \mathrm{e}-15 \\
1.1369 \mathrm{e}-17 \\
3.5527 \mathrm{e}-15 \\
1.8299 \mathrm{e}-14 \\
\end{array}$ \\
\hline Rastrigin & $\begin{array}{l}\text { Mean } \\
\text { Max } \\
\text { Median } \\
\text { Std.dev }\end{array}$ & $\begin{array}{l}13.77887 \\
15.03676 \\
8.399105 \\
1.507193 \\
\end{array}$ & $\begin{array}{l}\text { Mean } \\
\text { Max } \\
\text { Median } \\
\text { Std.dev }\end{array}$ & $\begin{array}{l}0 \\
0 \\
0 \\
0\end{array}$ & $\begin{array}{l}\text { Mean } \\
\text { Max } \\
\text { Median } \\
\text { Std.dev }\end{array}$ & $\begin{array}{c}2.6824 \mathrm{e}-11 \\
1.3337 \mathrm{e}-9 \\
2.3981 \mathrm{e}-14 \\
1.886 \mathrm{e}-10\end{array}$ & $\begin{array}{l}\text { Mean } \\
\text { Max } \\
\text { Median } \\
\text { Std.dev } \\
\end{array}$ & $\begin{array}{l}0 \\
0 \\
0 \\
0\end{array}$ \\
\hline Griewenk & $\begin{array}{l}\text { Mean } \\
\text { Max } \\
\text { Median } \\
\text { Std.dev } \\
\end{array}$ & $\begin{array}{c}0.057967 \\
13.054357 \\
0.023109 \\
0.016779 \\
\end{array}$ & $\begin{array}{l}\text { Mean } \\
\text { Max } \\
\text { Median } \\
\text { Std.dev }\end{array}$ & $\begin{array}{c}0.0039444 \\
0.019719 \\
2.8869 \mathrm{e}-18 \\
0.0052078 \\
\end{array}$ & $\begin{array}{l}\text { Mean } \\
\text { Max } \\
\text { Median } \\
\text { Std.dev }\end{array}$ & $\begin{array}{c}0.013861 \\
0.058867 \\
2.3981 \mathrm{e}-14 \\
0.01552 \\
\end{array}$ & $\begin{array}{l}\text { Mean } \\
\text { Max } \\
\text { Median } \\
\text { Std.dev }\end{array}$ & $\begin{array}{c}0.00025347 \\
0.0015353 \\
2.9696 \mathrm{e}-9 \\
0.00357468\end{array}$ \\
\hline Quartic & $\begin{array}{l}\text { Mean } \\
\text { Max } \\
\text { Median } \\
\text { Std.dev } \\
\end{array}$ & $\begin{array}{c}10.553674 \\
15.836762 \\
8.294792 \\
1.747389 \\
\end{array}$ & $\begin{array}{l}\text { Mean } \\
\text { Max } \\
\text { Median } \\
\text { Std.dev } \\
\end{array}$ & $\begin{array}{l}3.4938 \mathrm{e}-15 \\
8.1481 \mathrm{e}-16 \\
2.3981 \mathrm{e}-14 \\
1.1995 \mathrm{e}-15 \\
\end{array}$ & $\begin{array}{l}\text { Mean } \\
\text { Max } \\
\text { Median } \\
\text { Std.dev }\end{array}$ & $\begin{array}{l}3.1351 \mathrm{e}-17 \\
9.5804 \mathrm{e}-24 \\
2.5503 \mathrm{e}-21 \\
2.2243 \mathrm{e}-18 \\
\end{array}$ & $\begin{array}{l}\text { Mean } \\
\text { Max } \\
\text { Median } \\
\text { Std.dev } \\
\end{array}$ & $\begin{array}{c}1.5587 \mathrm{e}-28 \\
2.716 \mathrm{e}-27 \\
1.8532 \mathrm{e}-30 \\
4.035 \mathrm{e}-26 \\
\end{array}$ \\
\hline Spherical & $\begin{array}{l}\text { Mean } \\
\text { Max } \\
\text { Median } \\
\text { Std.dev }\end{array}$ & $\begin{array}{l}0.013378 \\
0.014291 \\
0.011198 \\
0.000905 \\
\end{array}$ & $\begin{array}{l}\text { Mean } \\
\text { Max } \\
\text { Median } \\
\text { Std.dev }\end{array}$ & $\begin{array}{l}3.9168 \mathrm{e}-16 \\
5.8856 \mathrm{e}-14 \\
9.3092 \mathrm{e}-19 \\
1.0291 \mathrm{e}-18 \\
\end{array}$ & $\begin{array}{l}\text { Mean } \\
\text { Max } \\
\text { Median } \\
\text { Std.dev }\end{array}$ & $\begin{array}{l}9.2696 \mathrm{e}-19 \\
4.9611 \mathrm{e}-17 \\
5.8252 \mathrm{e}-25 \\
8.6636 \mathrm{e}-21\end{array}$ & $\begin{array}{l}\text { Mean } \\
\text { Max } \\
\text { Median } \\
\text { Std.dev } \\
\end{array}$ & $\begin{array}{c}.3776 \mathrm{e}-28 \\
7.407 \mathrm{e}-27 \\
3.4564 \mathrm{e}-29 \\
1.2121 \mathrm{e}-27 \\
\end{array}$ \\
\hline
\end{tabular}

\section{CONCLUSION}

An approach for dealing with the stagnation problem in PSO has been tested by building into the algorithm a mechanism to automatically avoid premature convergence. The GCPSO mechanism helps liberate particles from the state of premature convergence and enables continued progress toward a global minimizer. PGCPSO has been shown to have better mean performance than the algorithms compared with a result that would have been more pronounced had only multi-modal bench- marks been used. PGCPSO also consistently outperformed in the presence of noise.

Given sufficient function evaluations, PGCPSO was able to solve the stagnation problem for each benchmark tested and approximate the true global minimizer with each trial conducted. Though the parameters used for PGCPSO worked consistently across the benchmark suite, it is not claimed that parameters have been fully optimized and it is not claimed that particles will not converge prematurely or itis absolutely free from stagnation. While PGCPSO seems capable of reducing the problem-dependency usually seen in the standard PSO algorithms so that parameteroptimization may be less important.

PGCPSO appears to be a good general purpose optimizer based on the benchmarks tested, which is certainly encouraging; however, it is cautioned that the empirical nature of the experiment is not a theoretical proof that PGCPSO will solve every problem well certainly, its performance must suffer somewhere. Future workwill try to understand where the algorithm suffers in order to understand any limitations and apply it to the proper contexts. This would allow eventual solution refinement of greater precision rather than repeatedly cutting of the local search in favor of exploration elsewhere. It has been empirically observed that clamping velocities to fifteen percent of the range of the search space on each dimension often provides a quicker convergence to

solutions of higher quality in conjunction with standard PSO. PGCPSO using standard Gbest PSO as its core, however, appearsto benefit from larger velocities such as those clamped tofiftypercent of the range on each dimension. The larger maximumvelocity facilitates exploration and moresignificant momentum by which to resist repeated premature convergence to the remembered global best.

PGCPSO seems to improve performance consistency with one set of parameters by facilitating escape from potentially deceitful local wells and to solve simple, unimodal problems free of entrapping wells quite well. It is suspected that PGCPSO may provide a degree of scalability previously missing in the standard PSO algorithm.

\section{REFERENCES}

[1] J. Kennedy and R. Eberhart.," Particle swarm optimization,". In Neural Networks, 1995. Proceedings., IEEE International Conference on, volume 4, 1995.

[2] F. Van den Bergh and A. P. Engelbrecht, "A new locally convergent particle swarm optimiser," in Proceedings of the IEEE Conference on Systems, Men, Cybernetics, ammamet, Tunisia, 2002, pp. 96-101.

[3] E.S. Peer, F. Van den Bergh, A.P. Engelbrecht, "Using neighbourhoods with the guaranteed convergence PSO" in Swarm Intelligence Symposium, 2003 Proceedings of the 2003 IEEE ,Digital Object Identifier: 10.1109/SIS.2003.1202274 Publication Year: 2003 , Page(s): $235-242$.

[4] G. Evers, "An automatic regrouping mechanism to deal with stagnation in particle swarm optimization," M.S. Thesis, 
Electrical Engineering Department, The University of TexasPan American, Edinburg, TX, 2009.

[5] Y. Shi and R. Eberhart.," A modified particle swarm optimizer". In Evolutionary Computation Proceedings, 1998. IEEE World Congress on Computational Intelligence., The 1998 IEEE International Conference on, pages 6973, 1998.

[6] Van den Bergh. ,An Analysis of Particle Swarm Optimizers". PhD thesis, University of Pretoria, Pretoria, 2002.
[7] M. Clerc and J. Kennedy. ,"The particle swarm-explosion, stability, and convergence in a multidimensional complex space". Evolutionary Computation, IEEE Transactions on, 6(1):5873, 2002.

[8] T. Bck. Evolutionary algorithms in theory and practice. Oxford University Press, 1996

[9] Test Functions for Unconstrained Global Optimization, Available:http://www.optimaampikyotou.ac.jp/member/stude nt/hedar/Hedarfiles/TestGOfiles/Page364.htm. 